\title{
Lattice QCD close to the light cone with applications to meson structure functions
}

\author{
D. Grünewald ${ }^{\star a}$, 'E.-M. Ilgenfritz ${ }^{b}$ and H.J. Pirner ${ }^{a c}$ \\ ${ }^{a}$ Institut für Theoretische Physik, Universität Heidelberg, Germany \\ ${ }^{b}$ Institut für Physik, Humboldt-Universität zu Berlin, Germany \\ ${ }^{c}$ Max-Planck-Institut für Kernphysik Heidelberg, Germany \\ E-mail: d.gruenewaldetphys . uni-heidelberg.de, \\ ilgenfriephysik.hu-berlin.de, \\ pirneretphys.uni-heidelberg.de
}

\begin{abstract}
We report on work in progress in which we calculate the gluon structure function of a color dipole in near light cone SU(2) lattice QCD. The color dipole is represented by a high (nearly infinite) momentum $q \bar{q}$ state added to the vacuum. We use as vacuum state a variational ground state of the near light cone Hamiltonian. We compute the color dipole matrix element of two transverse gluon momentum operators separated along the light cone, the Fourier transform of which is the gluon structure function. We identify three different contributions which, for large light cone momentum, reproduce the Pomeron behavior of the structure function with unit intercept.
\end{abstract}

LIGHT CONE 2008 Relativistic Nuclear and Particle Physics

July 7-11 2008

Mulhouse, France

\footnotetext{
* Speaker.

${ }^{\dagger}$ D. G. acknowledges funding by the European Union project EU RII3-CT-2004-506078 and the GSI Darmstadt.
} 


\section{Introduction}

The first support for our understanding of QCD as the theory of strong interactions has come from deep inelastic scattering. The structure of the proton unfolds itself as partons that experience only interactions small in strength due to asymptotic freedom. The so obtained structure functions representing the proton constituents can be considered as Fourier transforms of quark/gluon operators separated along light like distances. Since the discovery of scaling in deep inelastic scattering, calculations of perturbative corrections have strongly contributed to the credibility of QCD. For small $x_{B}$, there appear other contributions like $\alpha_{s}\left(Q^{2}\right) \log \left(1 / x_{B}\right)$ in addition to terms from DGLAP-evolution which need special care. It must be stressed that in a perturbative framework structure functions themselves cannot be calculated. Euclidean lattice simulations use the operator product expansion to get information about structure functions. In this way, the lowest moments of the pion and nucleon structure functions have been simulated [1,2]. Nowadays, even moments of generalized parton distributions are being calculated [3]. Recently, loop-loop correlation functions of tilted Wegner-Wilson loops have been computed on a Euclidean lattice [4] which can be related to the gluon distribution function $[5,6,7]$ of a color dipole or a hadron, in principle.

Independently of these investigations, the light cone lattice community has pursued [8] the idea of a different formulation of QCD on or near the light cone. The hope has been that a theoretical framework based on constituents moving along the light cone will be simple, following the experimental discovery of quarks rather closely. Of course, the light cone approach must attempt to incorporate the nonperturbative QCD vacuum, which is rather difficult to represent in terms of a Fock representation of free fields acting on a trivial vacuum. Also rewriting a spatially quantised theory into a theory quantised on a light like surface may cause problems.

Therefore, in a recent paper [9] we have advocated a near light cone (nlc) approach in which we strive to combine the advantages of the lattice world with the advantages of light cone simplifications. Here, "near to the light cone" refers to the employment of near light cone coordinates $[10,11]$ which have been initially introduced in order to investigate light front quantisation as a limiting procedure of equal time quantised theories. These coordinates depend on the external parameter $\eta$ which controls the distance to the light cone. In Ref. [9] we have found a ground state of the near light cone Hamiltonian which, in the light cone limit, is simpler than the ground state in equal time Hamiltonian theory. In the following, we report on work in progress, in which we use this variationally optimized ground state wave functional to determine the full gluon distribution function of a color dipole state, i.e. without the restriction to the computation of moments, as a first application.

\section{Definition of the color dipole and the gluon matrix element}

In deep inelastic scattering the hadronic target is probed on the light cone, i.e. at equal light cone time $x^{+}=0$. Here, $x^{+}=\left(x^{0}+x^{3}\right) / \sqrt{2}$ and $x^{-}=\left(x^{0}-x^{3}\right) / \sqrt{2}$ are the light cone temporal and longitudinal coordinate where $x^{0}, x^{3}$ denote the ordinary Minkowski coordinates in the laboratory frame. In light cone quantisation one quantises on exactly such a hypersurface, i.e. on a hypersurface defined by $x^{+}=0$. This has the advantage that one does not need to evolve the hadronic wave function during the scattering process in light cone time. We analyse the gluonic structure of 
a dipole state of a fixed transversal extension $\vec{d}_{\perp}$ in the following. We start from a dipole localized in configuration space consisting of a valence quark at $\left(x^{-}, \vec{x}_{\perp, q}=-\vec{d}_{\perp} / 2\right)$, a valence antiquark at $\left(x^{-}, \vec{x}_{\perp, \bar{q}}=\vec{d}_{\perp} / 2\right)$ and a gluon cloud represented by a Schwinger string $S_{\perp}\left(\vec{x}_{\perp, q}, \vec{x}_{\perp, \bar{q}} ; x^{-}\right)$connecting the quark and antiquark along the transversal direction which ensures gauge invariance. This state is projected onto a fixed center of mass momentum $p_{-}, \vec{p}_{\perp}$ which means that one has to integrate over all possible translations of this state decorated by the appropriate phase factor $e^{-\mathrm{i} p_{-} x^{-}}$. The gluon distribution function $f_{g / d}\left(x_{B}\right)$ gives the probability that a gluon carries the longitudinal momentum fraction $x_{B}$ of the fast moving dipole $d$ [12]. It is given by the Fourier transformation of the correlation function of longitudinally separated transversal gluon electric field strength operators connected by a straight Schwinger string $S_{a b}^{A}$ in the adjoint representation and normalized to the longitudinal momentum $p_{-}$of the target

$$
f_{g / d}\left(x_{B}\right)=\frac{1}{x_{B}} \frac{1}{2 \pi} \int d z^{-} e^{-\mathrm{i} x_{B} p_{-} z^{-}} \frac{1}{p_{-}}\left\langle d\left(p_{-}, \vec{d}_{\perp}\right)\left|G^{++}\left(z^{-}, 0\right)\right| d\left(p_{-}, \vec{d}_{\perp}\right)\right\rangle_{c} .
$$

The index "c" implies to take the connected matrix element and $\left|d\left(p_{-}, \vec{d}_{\perp}\right)\right\rangle$ denotes the dipole state. In the light cone limit $(\eta \rightarrow 0)$ of the near light cone Hamiltonian, the gluon electric field strength operators are given by the corresponding momentum operators.

$$
\begin{gathered}
G^{++}\left(z^{-}, 0\right)=\sum_{k} \Pi_{k}^{a}\left(z^{-}, \overrightarrow{0}_{\perp}\right) S_{a b}^{A}\left(z^{-}, 0 ; \overrightarrow{0}_{\perp}\right) \Pi_{k}^{b}\left(0, \overrightarrow{0}_{\perp}\right),\left[\Pi_{k}^{a}(\vec{x}), A_{l}^{b}(\vec{y})\right]=-\mathrm{i} \delta^{(3)}(\vec{x}-\vec{y}) \delta_{k, l} \delta^{a, b} \\
S_{a b}^{A}\left(z^{-}, 0 ; \overrightarrow{0}_{\perp}\right)=\left[\mathscr{P} \exp \left\{\mathrm{i} g \int_{0}^{z^{-}} d v^{-} A_{-}^{c}\left(v^{-}, \overrightarrow{0}_{\perp}\right) \lambda_{a d j}^{c}\right\}\right]_{a b} .
\end{gathered}
$$

The gluon electric field strength operators do not commute with the transverse strings in the dipole wave functions as shown in Eq. (2.2). Therefore the string $S_{\perp}^{\dagger}\left(\vec{x}_{q}, \vec{x}_{\bar{q}} ; y^{-}\right)$arising from the links in the outgoing dipole at $y^{-}$must appear to the left of the operator $G^{++}$and correspondingly the string $S_{\perp}\left(\vec{x}_{q}, \vec{x}_{\bar{q}} ; x^{-}\right)$in the incoming dipole at $x^{-}$to the right of $G^{++}$(see Fig. 1). Then the matrix element with the nlc ground state $\left|\Psi_{0}\right\rangle$ is written

$$
\begin{aligned}
& \left\langle d\left(p_{-}, \vec{d}_{\perp}\right)\left|G^{++}\left(z^{-}, 0\right)\right| d\left(p_{-}, \vec{d}_{\perp}\right)\right\rangle=\frac{2 p_{-} V}{N} \int d x^{-} d y^{-} d^{2} x_{\perp} e^{-\mathrm{i} p_{-}\left(x^{-}-y^{-}\right)} \\
& \cdot\left\langle\Psi_{0}\left|\frac{1}{2} \operatorname{Tr}\left[S_{-}^{\dagger}\left(y^{-}, x^{-} ; \vec{x}_{\bar{q}}\right) S_{\perp}^{\dagger}\left(\vec{x}_{q}, \vec{x}_{\bar{q}} ; y^{-}\right) S_{-}\left(y^{-}, x^{-} ; \vec{x}_{q}\right) G^{++}\left(z^{-}, 0\right) S_{\perp}\left(\vec{x}_{q}, \vec{x}_{\bar{q}} ; x^{-}\right)\right]\right| \Psi_{0}\right\rangle .
\end{aligned}
$$

At high momentum, the quark and antiquark in the color dipole move on straight line classical trajectories where they pick up non-abelian phase factors along their paths. Thereby the overlap of the dipole state $\left|d\left(p_{-}, \vec{d}_{\perp}\right)\right\rangle$ with itself is related to the matrix element of a Wegner-Wilson loop in a pure gauge vacuum field configurations. Let $S_{-}, S_{-}^{\dagger}$ connect the end points of the color dipoles along the $x^{-}$- direction. Then the eikonal trajectories of the quark and antiquark (dotted lines) together with the strings (full lines) connecting the quark and antiquark in the color dipole constitute the contour of the Wegner-Wilson loop W (shown in Fig. 1):

$$
W\left(x^{-}, y^{-} ; \vec{x}_{q}, \vec{x}_{\bar{q}}\right)=S_{-}^{\dagger}\left(y^{-}, x^{-} ; \vec{x}_{\bar{q}}\right) S_{\perp}^{\dagger}\left(\vec{x}_{q}, \vec{x}_{\bar{q}} ; y^{-}\right) S_{-}\left(y^{-}, x^{-} ; \vec{x}_{q}\right) S_{\perp}\left(\vec{x}_{q}, \vec{x}_{\bar{q}} ; x^{-}\right) .
$$

The constant $N$ is determined in such a way that the dipole state satisfies the boost invariant normalization $\left\langle d\left(p_{-}, \vec{d}_{\perp}\right) \mid d\left(p_{-}, \vec{d}_{\perp}\right)\right\rangle=2 p_{-} V$, where the volume is $V=L_{-} L_{\perp}^{2}$. 


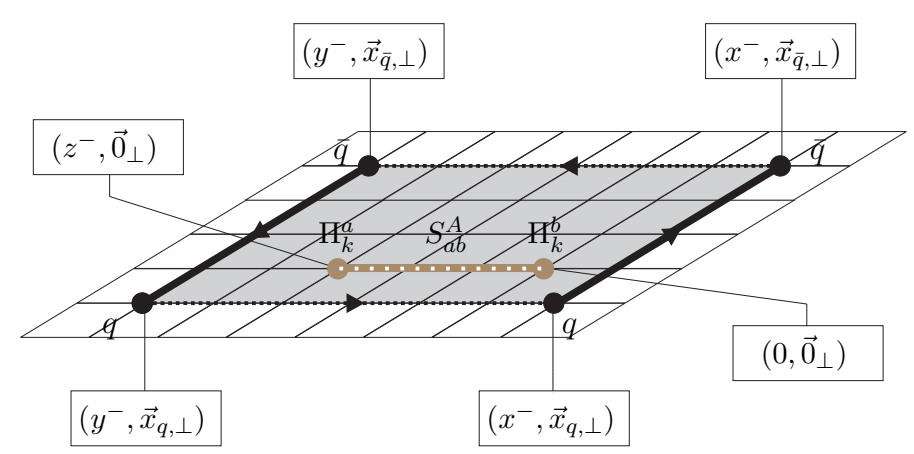

Figure 1: Graphical representation of the Wilson loop mapped out by the color dipole with a quark $q$ and an antiquark $\bar{q}$ moving along the $x_{-}$direction. The full curve gives the strings which connect the quark and antiquark in the dipole states. The dotted strings arise due to the elimination of the quark spinors using the eikonal approximation of the quark propagator. The dash dotted insertion in the so formed Wegner-Wilson loop represents the gluon momentum correlation function.

\section{Lattice calculation}

Having defined the model in the continuum in the last section, we calculate the gluon distribution function using the variationally optimized ground state wave functional Eq. (3.1) (below) of the near light cone (nlc) lattice Hamiltonian in the pure gauge sector now (for details concerning the nlc Hamiltonian and its variationally optimized ground state wave functional c.f. Ref [9]). The effective nlc lattice Hamiltonian and a variationally optimized ground state wave functional $\left|\Psi_{0}\right\rangle$ have been determined in ref. [9]. In our Hamiltonian approach, we stay in Minkowski time, i.e. a Wick rotation to imaginary nlc times is not necessary. The wave functional contains a product of single site plaquettes $U_{-k}(\vec{x})$ and $U_{12}(\vec{x})$ with two variationally optimized parameters $\rho_{0}$ and $\delta_{0}$ which are functions of the lattice coupling $\lambda=4 / g^{4}$ related to the gauge coupling $g$ and the distance to the light cone $\eta$ normalized by $N_{\Psi}$

$$
\begin{gathered}
\left|\Psi_{0}\right\rangle=\sqrt{N_{\Psi}} e^{f[U]}|0\rangle \\
f[U]=\sum_{\vec{x}}\left\{\sum_{k=1}^{2} \rho_{0}(\lambda, \eta) \operatorname{Tr}\left[\operatorname{Re}\left(U_{-k}(\vec{x})\right)\right]+\delta_{0}(\lambda, \eta) \operatorname{Tr}\left[\operatorname{Re}\left(U_{12}(\vec{x})\right)\right]\right\}|0\rangle, \\
U_{i j}(\vec{x})=U_{i}(\vec{x}) U_{j}\left(\vec{x}+\vec{e}_{i}\right) U_{i}^{\dagger}\left(\vec{x}+\vec{e}_{j}\right) U_{j}^{\dagger}(\vec{x}), U_{j}(\vec{x}) \equiv \mathscr{P} \exp \left(\mathrm{i} g \int_{\vec{x}}^{\vec{x}+\widehat{e}_{j}} d y^{\mu} A_{\mu}^{a}(y) \lambda^{a}\right) .
\end{gathered}
$$

Here, $\lambda^{a}$ represent the generators of $S U(2), \widehat{e}_{i}$ is the unit vector in direction $i=1,2,-$ and the state $|0\rangle$ is given by the trivial ground state which is annihilated by the lattice momenta $\widehat{\Pi}_{k}^{a}(\vec{x})$ (here and in the following quantities in lattice units are indicated by a hat) canonically conjugate to the links $U_{j}(\vec{y})$,

$$
\widehat{\Pi}_{k}^{a}(\vec{x})|0\rangle=0 \text { and }\langle 0| \widehat{\Pi}_{k}^{a}(\vec{x})=0 \forall \vec{x}, k, a \quad, \quad\left[\widehat{\Pi}_{i}^{a}(\vec{x}), U_{j}(\vec{y})\right]=\lambda^{a} U_{i}(\vec{x}) \delta_{\vec{x}, \vec{y}} \delta_{i, j} .
$$

This ground state wave functional is similar to the ground state wave functional used in equal time quantised lattice gauge theory [14]. However, it allows for an asymmetry in the gauge links dynamics in the purely transversal and the transversal longitudinal plane rendering the correspondent 
asymmetry in the Hamiltonian. Similar to the equal time case, it does not allow for a transition from the discrete lattice to the continuum due to the lack of a scaling regime. We have optimized this ansatz over a large region in $(\lambda, \eta)$-space which allows us to extrapolate the parameters $\rho_{0}, \delta_{0}$ to the light cone $\eta \rightarrow 0$. This limit yields the following functional behavior

$$
\rho_{0}(\lambda, 0)=\left(0.65-\frac{0.87}{\lambda}+\frac{1.65}{\lambda^{2}}\right) \sqrt{\lambda}, \delta_{0}(\lambda, 0)=\left(0.05+\frac{0.04}{\lambda}-\frac{1.39}{\lambda^{2}}\right) \sqrt{\lambda} .
$$

Note that the ground state Eq. (3.1) is an approximation of the fully interacting effective Hamiltonian and does not rely on any truncated Fock space expansion around the perturbative vacuum. In order to transcribe the continuum definition of the gluon distribution function Eq. (2.1) to the lattice, we replace all integrations by sums over lattice sites times the correspondent lattice spacing. To compute the gluon matrix elements one has to commute the momentum operators to the left or to the right until they stand directly in front of the trivial ground "ket" state $|0\rangle$ or the corresponding "bra" state $\langle 0|$ which they annihilate (cf. Eq. (3.3)). Then, the matrix elements do only depend on the links which means that they can be evaluated by standard methods. (In the following, we assume that $\vec{d}_{\perp}$ is parallel to one of the transversal coordinate axes, i.e. $\vec{d}_{\perp}=\left|\widehat{d}_{\perp}\right| \cdot \vec{e}_{k}$ with $k=1$ ).

For the evaluation of the matrix elements discussed in section 2 the wave functional $\left|\Psi_{0}\right\rangle$ plays the essential role. The gauge dynamics in the transverse $(1,2)$ plane is strongly coupled as shown by the parameter $\delta_{0}(\lambda, 0) \approx 0$ (cf. Eq. (3.4)). A strong coupling approximation Eq. (3.5) turns out to be valid even for values of $\lambda$ which are far beyond $\lambda<<1$, i.e. $\lambda=10$ as proven by actual Monte Carlo sampling of the ground state wave functional Eq. (3.1) [15]. In the light cone limit $\eta \rightarrow 0$ the gauge dynamics in each of the hyperplanes $(-, 1)$ and $(-, 2)$ is essentially two dimensional because of the small coupling of their dynamics via the gauge fluctuations in the $(1,2)$ plane. In two dimensions with free boundary conditions the strong coupling approximation is exact. It is here where we see an important simplification of the gluon dynamics on the light cone compared with equal time Hamiltonian QCD. Under these conditions, we have the following standard area law behavior for Wegner-Wilson loops in the $(-, k)$ directions

$$
\left\langle\Psi_{0}\left|\frac{1}{2} \operatorname{Tr}\left[W\left(0, \widehat{z}^{-} ; 0, d_{\perp}\right)\right]\right| \Psi_{0}\right\rangle=\left(\left\langle\Psi_{0}\left|\frac{1}{2} \operatorname{Tr}\left[U_{-k}\right]\right| \Psi_{0}\right\rangle\right)^{\widehat{d}_{\perp}|\widehat{z}|} \quad, \text { area }=\widehat{d_{\perp}}\left|\widehat{z}^{-}\right| a_{\perp} a_{-} .
$$

Factorization is also true for expectation values of the product of two Wegner-Wilson loops which do not overlap. Single plaquette expectation values with respect to the ground state wave functional are given by

$$
f_{1 k} \equiv\left\langle\Psi_{0}\left|\frac{1}{2} \operatorname{Tr}\left[U_{-k}\right]\right| \Psi_{0}\right\rangle=\frac{I_{2}\left(4 \rho_{0}\right)}{I_{1}\left(4 \rho_{0}\right)}+\mathscr{O}\left(\delta_{0}^{2}\right) \in[-1,1]
$$

Here, $I_{n}$ denote the modified Bessel functions of the first kind. One obtains three contributions $<d\left|\widehat{G}_{i}^{++}\left(\widehat{z}^{-}, 0\right)\right| d>_{c}$ with $i=1,2,3$ to the matrix element of $<d\left|\widehat{G}^{++}\left(\widehat{z}^{-}, 0\right)\right| d>_{c}$. The first contribution is given by the application ${ }^{1}$ of both of the momentum operators of the correlation function Eq. (2.2) onto either the transversal Schwinger string of the incoming or the outgoing dipole state. Hence, this matrix element is given by

$$
\frac{1}{\widehat{p}_{-}}<d\left|\widehat{G}_{1}^{++}\left(\widehat{z}^{-}\right)\right| d>=\frac{3}{4} \widehat{d}_{\perp} \delta_{\widehat{z}^{-}, 0} .
$$

\footnotetext{
${ }^{1}$ Here, $\widehat{\Pi}_{k}$ applied onto an operator means to take the commutator of $\widehat{\Pi}_{k}$ with this operator
} 
It is proportional to the transversal extension of the dipole and to the Casimir operator of the fundamental representation. It has a non-vanishing support only at zero separation $\widehat{z}^{-}=0$, i.e. for overlapping gluon operators. For $\widehat{z}^{-}=0$ the operator $\widehat{G}^{++}(0)$ represents the gluonic longitudinal momentum density operator $T^{++}(0)$ at the origin $\left(0, \overrightarrow{0}_{\perp}\right)$. Therefore this contribution is associated with the number of gluons in the flux tube of the color dipole which increases linearly with the length of the tube. Beside this trivial dependence on $\widehat{d}_{\perp}$, this matrix element is extremely localized in configuration space, i.e. its Fourier transformation is uniformly distributed in $x_{B}$.

The second matrix element $\left\langle d\left|G_{2}^{++}\right| d\right\rangle / \widehat{p}_{-}$is given by the application of the first momentum operator of the correlation function Eq. (2.2) onto the transversal Schwinger string of the incoming dipole state and the application of the second momentum operator onto the transversal Schwinger string of the outgoing dipole state or vice versa. This matrix element reads

$$
\frac{1}{\widehat{p}_{-}}<d\left|\widehat{G}_{2}^{++}(\widehat{z})\right| d>=\frac{3}{4} \widehat{d}_{\perp} \cos \left(\widehat{p}_{-} \widehat{z}^{-}\right) f_{1 k}^{\widehat{d}_{\perp}|\widehat{z}|} /\left(\sum_{\widehat{x}^{-}} \cos \left(\widehat{p}_{-} \widehat{x}\right)\left(f_{1 k}\right)^{\widehat{d}_{\perp}|\widehat{x}|}\right) .
$$

Here, the denominator represents the normalization of the hadronic state $N / \hat{V}$. The numerator is again proportional to the transversal extension of the dipole and to the Casimir operator of the fundamental representation. The application of the gluon momentum operators bring the incoming and outgoing color dipole states into the adjoint representation, i.e. they represent excited states. The adjoint Schwinger string which ensures gauge invariance of this excited state corresponds to a gluon propagating along the light cone in the eikonal approximation. The generated excited state is equivalent to a state with two dipoles which accounts for hadronisation. We study two dipoles sizes: $\left(\widehat{d}_{\perp}=5\right.$ and $\left.\widehat{d_{\perp}}=30\right)$. Its behavior is determined by the area law behavior of the WegnerWilson loop. For small $\widehat{d}_{\perp}$, the Wegner-Wilson loop decreases slowly as a function of $\widehat{z}^{-}$. For large values of $\widehat{d}_{\perp}$, correlation functions at non-vanishing $\widehat{z}^{-}$are highly suppressed and one recovers the extremely localized behavior of the first matrix element, i.e. a Kronecker delta in $\widehat{z}^{-}$. Hence, after the Fourier transformation this contribution to the gluon distribution function is also flat for large $\widehat{d_{\perp}}$.

The third part of the matrix element is the vacuum fluctuation term which originates from the application of the momentum operators of the correlation function Eq. (2.2) onto the ground state wave functional Eq. (3.1). Its magnitude is considerably smaller than the magnitudes of the first two contributions. This is due to the fact, that the connected matrix element subtracts exactly these kinds of vacuum to vacuum transitions. Hence, this part of the matrix element can be neglected.

An interpretation of our results has to be qualitative since our wave functional does not allow a continuum limit of vanishing lattice size. If we use a transverse lattice size equal to the resolution of the probe $a_{\perp}=1 / Q$ where $\mathrm{Q}$ is the scale of the gluon structure function, then one sees that increasing resolution at fixed physical dipole size $d$ generates a strongly localized correlation function of gluon field strengths along the light cone. This feature yields a gluon structure function of the dipole which behaves like the Pomeron structure function with unit intercept, i.e.

$$
\lim _{Q|\vec{d}| \rightarrow \infty} f_{g / d}\left(x_{B}, Q^{2}\right) \sim \frac{1}{x_{B}} .
$$

Modeling the hadron as a simple dipole of fixed size $d$ without any detailed quark-antiquark wave function, one cannot expect a meaningful gluon structure function for large $x_{B}$. At large values 
of $x_{B}$, the gluon distribution function has to decrease with the number of spectator partons [16]. But, the gluon in our model does not know about the spectator $q$ and $\bar{q} x_{B}$-distributions, since they are not yet implemented in our simple approach. In a forthcoming paper however, we model the quark/antiquark distributions explicitly.

Since the ground state wave functional ansatz Eq. (3.1) does not provide a well defined continuum limit the exact prefactor of our calculations cannot be trusted. There have been indications for scaling solutions for equal time Hamiltonian QCD in a guided Greens function Monte-Carlo sampling of the ground state probability density [17]. The ground state wave functional used in that reference includes extended plaquette terms similar to improved actions in Euclidean lattice approaches. In ref. [15] the vacuum state in the light cone limit is exactly given by a weighted sum of plaquette plaquette correlations separated along the spatial light cone coordinate. This vacuum will give a dressed dipole state which contains fluctuations of the transverse string in the dipole which lead to an additional cross section at small $x_{B}$. Ultimately the $q \bar{q}$ ground state is given by applying the imaginary time evolution operator $\exp \left(-P_{+} x^{+}\right)$on the dipole in the limit $x^{+} \rightarrow \infty$. This means that the world sheet swept out by the color dipole in the ground state will not be only given by a rectangular Wegner-Wilson loop. As said the transversal Schwinger strings which follow straight lines in the strong coupling approximation are washed out and yield a more subtle structure of the gluon distribution functions than the Pomeron behavior.

\section{References}

[1] M. Gockeler, R. Horsley, D. Pleiter, P. E. L. Rakow and G. Schierholz [QCDSF Collaboration], Phys. Rev. D 71 (2005) 114511 [arXiv:hep-ph/0410187].

[2] J. W. Negele et al., Nucl. Phys. Proc. Suppl. 128 (2004) 170 [arXiv:hep-lat/0404005].

[3] P. Hagler, J. W. Negele, D. B. Renner, W. Schroers, T. Lippert and K. Schilling [LHPC collaboration and SESAM collaboration], Phys. Rev. D 68 (2003) 034505 [arXiv:hep-lat/0304018].

[4] M. Giordano and E. Meggiolaro, arXiv:0808.1022 [hep-lat].

[5] N. N. Nikolaev and B. G. Zakharov, Z. Phys. C 49 (1991) 607.

[6] K. J. Golec-Biernat and M. Wusthoff, Phys. Rev. D 60 (1999) 114023 [arXiv:hep-ph/9903358].

[7] A. I. Shoshi, F. D. Steffen, H. G. Dosch and H. J. Pirner, Phys. Rev. D 66 (2002) 094019 [arXiv:hep-ph/0207287].

[8] S. Dalley, "Light cone physics: Hadrons and beyond: Proceedings. 2003".

[9] D. Grunewald, E. M. Ilgenfritz, E. V. Prokhvatilov and H. J. Pirner, Phys. Rev. D 77 (2008) 014512 [arXiv:0711.0620 [hep-lat]].

[10] E. V. Prokhvatilov and V. A. Franke, Sov. J. Nucl. Phys. 49 (1989) 688 [Yad. Fiz. 49 (1989) 1109].

[11] F. Lenz, H. W. L. Naus and M. Thies, Annals Phys. 233 (1994) 317.

[12] J. C. Collins and D. E. Soper, Nucl. Phys. B 194 (1982) 445.

[13] A. I. Shoshi, F. D. Steffen, H. G. Dosch and H. J. Pirner, Phys. Rev. D 68 (2003) 074004 [arXiv:hep-ph/0211287].

[14] S. A. Chin, O. S. Van Roosmalen, E. A. Umland and S. E. Koonin, Phys. Rev. D 31 (1985) 3201. 
[15] D. Grünewald, Phd. thesis, University of Heidelberg, Universitätsbibliothek Heidelberg, http://www.ub.uni-heidelberg.de/archiv/8601/.

[16] R. Blankenbecler and S. J. Brodsky, Phys. Rev. D 10 (1974) 2973.

[17] M. Beccaria, Phys. Rev. D 62 (2000) 034510 [arXiv:hep-lat/0003016]. 